\title{
Masked inherited primary arrhythmia syndromes in sudden cardiac death patients accompanied by coronary vasospasm
}

Ki Hong Lee ${ }^{1}$, Hyung Wook Park ${ }^{1}$, Jeong Nam Eun ${ }^{1}$, Jeong Gwan Cho' , Nam Sik Yoon ${ }^{1}$, Mi Ran Kim², Yo Han $\mathrm{Ku}^{1}$, Hyukjin Park ${ }^{1}$, Seung Hun Lee ${ }^{1}$, Jeong Han Kim ${ }^{1}$, Min Chul Kim ${ }^{1}$, Woo Jin Kim ${ }^{1}$, Hyun Kuk Kim ${ }^{1}$, Jae Yeong Cho ${ }^{1}$, Keun-Ho Park ${ }^{1}$, Doo Sun Sim ${ }^{1}$, Hyun Ju Yoon ${ }^{1}$, Kye Hun Kim ${ }^{1}$, Young Joon Hong ${ }^{1}$, Ju Han Kim ${ }^{1}$, Youngkeun Ahn ${ }^{1}$, Myung Ho Jeong ${ }^{1}$, and Jong Chun Park ${ }^{1}$

${ }^{1}$ Department of Cardiovascular Medicine, Chonnam National University Hospital, Gwangju; ${ }^{2}$ Department of Nursing, Chunnam Techno University, Gokseong, Korea

Received: August 20, 2015

Revised : December 1, 2015

Accepted: December 6, 2015

\section{Correspondence to}

Hyung Wook Park, M.D.

Department of Cardiovascular

Medicine, The Heart Center of

Chonnam National University

Hospital, 42 Jebong-ro, Dong-gu,

Gwangju 61469, Korea

Tel: +82-62-220-6572

Fax: +82-62-223-3105

E-mail: mdhwp@chol.com
Background/Aims: Coronary vasospasms are one of the important causes of sudden cardiac death (SCD). Provocation of coronary vasospasms can be useful, though some results may lead to false positives, with patients potentially experiencing recurrent SCD despite appropriate medical treatments. We hypothesized that it is not coronary vasospasms but inherited primary arrhythmia syndromes (IPAS) that underlie the development of SCD.

Methods: We analyzed 74 consecutive patients (3.8\%) who survived out-of-hospital cardiac arrest among 1,986 patients who had angiographically proven coronary vasospasms. Electrical abnormalities were evaluated in serial follow-up electrocardiograms (ECGs) during and after the index event for a 3.9 years median follow-up. Major clinical events were defined as the composite of death and recurrent SCD events. Results: Forty five patients (6o.8\%) displayed electrocardiographic abnormalities suggesting IPAS: Brugada type patterns in six (8.2\%), arrhythmogenic right ventricular dysplasia patterns in three (4.1\%), long QT syndrome pattern in one (2.2\%), and early repolarization in 38 (51.4\%). Patients having major clinical events showed more frequent Brugada type patterns, early repolarization, and more diffuse multivessel coronary vasospasms. Brugada type pattern ECGs (adjusted hazard ratio [HR], 4.22; 95\% confidence interval [CI], 1.16 to 15.99; $p=0.034$ ), and early repolarization (HR, 2.97; 95\% CI, 1.09 to 8.10; $p=0.034$ ) were ultimately associated with an increased risk of mortality.

Conclusions: Even though a number of aborted SCD survivors have coronary vasospasms, some also have IPAS, which has the potential to cause SCD. Therefore, meticulous evaluations and follow-ups for IPAS are required in those patients.

Keywords: Heart arrest; Coronary vasospasm; Inherited arrhythmia

\section{INTRODUCTION}

Lethal ventricular arrhythmia can occur in patients having no structural heart disease [1]. Coronary vasospasms or inherited primary arrhythmia syndromes (IPAS) are leading causes of sudden catastrophes [2-5]. Coronary vasospasms have been known to induce ventricular arrhythmia in patients without the presence of obstruct- 
ing coronary artery lesions, and even without chest pain [2]. In addition, some patients experience recurrent ventricular arrhythmias or sudden cardiac death (SCD) despite treatment using anti-spastic medication (e.g., calcium channel blockers, nitrates) $[6,7]$. However, the reason for why lethal ventricular arrhythmia develops in patients having coronary vasospasms is not fully understood. Few studies have reported on the abnormalities of ventricular repolarization, even during asymptomatic phases of their disease, or had increased QT dispersion $[8,9]$.

Brugada syndrome, arrhythmogenic right ventricular dysplasia (ARVD), and long or short QT syndrome are well-known IPAS and are often the cause of SCD in a structurally normal heart [3-5,10]. Early repolarization, which has been considered as a benign electrocardiogram (ECG) manifestation, has also been associated with ventricular fibrillation and SCD [11]. However, because the ECG abnormalities of IPAS change spontaneously day-to-day, IPAS might be hidden or masked during the index event. Therefore, we hypothesize here that IPAS might be masked in SCD in patients having a coronary vasospasm.

\section{METHODS}

\section{Study population}

Seventy-four aborted SCD patients (3.8\%) were analyzed retrospectively among 1,986 patients who displayed a coronary vasospasm on a coronary angiogram from January 2003 to July 2012. Of these 1,986 patients, 173 experienced SCD events (8.7\%). Exclusion criteria included patients who had $\geq 50 \%$ obstructive coronary artery stenosis $(\mathrm{n}=80,4.0 \%)$, coexisting IPAS (Brugada syndrome, $\mathrm{n}=7,0.4 \%$; long-QT syndrome, $\mathrm{n}=6,0.3 \%$ ), and structural heart disease (dilated cardiomyopathy, $\mathrm{n}=2,0.1 \%$; hypertrophic cardiomyopathy, $\mathrm{n}=1,0.1 \%$; atrioventricular block, $n=3,0.2 \%)$. Finally, 74 SCD patients associated with coronary vasospasm were analyzed in this study.

Coronary vasospasm was defined as the condition in which the coronary angiography showed no significant obstructive coronary artery lesion (stenosis $<50 \%$ ) and the occurrence of transient or near total obstruction (> $90 \%$ ) by either spontaneous coronary spasms or an intracoronary ergonovine provocation induced vasospasm that can be relieved by the intracoronary injection of ni- troglycerin.

The population was classified into group I (ECG abnormalities suggesting IPAS, $\mathrm{n}=45$ ) and group II (no ECG abnormalities suggesting IPAS, $n=29$ ). Further classifications included group III (patients having major clinical events, $\mathrm{n}=26$ ) and group IV (patients not having major clinical events, $n=48$ ) according to clinical outcomes. Major clinical events were defined as the composite of death and recurrent SCD events. Electrocardiographic findings, demographic features, angiographic results, and concomitant medications were analyzed in all of the eligible patients during a 3.9-year (range, 2.2 to 6.5) median follow-up period. All patients gave written informed consent before coronary angiogram and ergonovine provocation tests.

\section{Coronary angiography and ergonovine provocation test}

Coronary angiography and intracoronary ergonovine provocation tests were performed through the femoral artery using a standard technique. All cardiovascular medicines that could affect angiogram results were discontinued for more than three times their half-lives. Ergonovine was infused in a stepwise manner into the left coronary artery $(20$ to $100 \mu \mathrm{g})$ and the right coronary artery $(20$ to $50 \mu \mathrm{g})$ over 20 seconds, with 3 to 5 minutes intervals between each injection. Repeated angiograms were performed 90 seconds after each injection.

\section{Electrocardiographic analysis}

All 12-lead ECGs were recorded at the time of the SCD event, and during follow-up were reviewed by two electrophysiologists that were blinded to the clinical outcomes. Brugada type ECG diagnoses were made according to the previous definition [12]. The type 1 Brugada ECG was defined as a coved ST-segment elevation $\geq 2$ $\mathrm{mm}(0.2 \mathrm{mV})$ followed by a negative $\mathrm{T}$ wave in the $>1$ right precordial lead ( $\left.\mathrm{V}_{1}-\mathrm{V}_{3}\right)$. The type 2 Brugada ECG was characterized by a saddle back appearance having a ST-segment elevation of $\geq 2 \mathrm{~mm}$ with a trough of $\geq$ $1 \mathrm{~mm}$ followed by a positive or biphasic $\mathrm{T}$ waves. The type 3 Brugada ECG was defined as having a saddle-back appearance and a ST-segment elevation of $\geq 2 \mathrm{~mm}$ with a trough of $<1 \mathrm{~mm}$ followed by a positive $\mathrm{T}$ wave. The diagnosis of ARVD included a $\mathrm{T}$ wave inversion, a prolonged QRS > $110 \mathrm{~ms}$ in the right precordial leads, or the presence of an epsilon wave [13]. Long QT syndrome 
(LQTS) was defined in high probability patients according to the consensus diagnostic criteria [12].

The electrocardiographic diagnosis of early repolarization should have a $\mathrm{J}$ point elevation $\geq 0.1 \mathrm{mV}$ in at least two inferior or lateral leads. Notched-type repolarization was defined as a positive J deflection at the end of the QRS complex, and slurred-type repolarization had a terminal slower wave from the QRS J point to the ST segment. ST segment patterns after the J point elevation were classified as concave/rapidly ascending (> 0.1 $\mathrm{mV}$ elevation of ST segment within $100 \mathrm{~ms}$ after the J point, or a persistently elevated ST segment of $>0.1 \mathrm{mV}$ throughout the ST segment) or horizontal/descending ( $\leq$ o.1 $\mathrm{mV}$ elevation of the ST segment within $100 \mathrm{~ms}$ after the J point). The isoelectric line (baseline) was defined as the level between two T-P segments $[12,14]$.

\section{Statistical analysis}

All statistical analyses were performed with SPSS version 18.0 (SPSS Inc., Chicago, IL, USA). For continuous variables, differences between groups were evaluated using an unpaired $t$ test or Mann-Whitney rank-sum test. For discrete variables, differences were expressed as counts and percentages, and were analyzed using a chi-square test (or Fisher exact test) between groups, as appropriate. We constructed Kaplan-Meier curves to de-

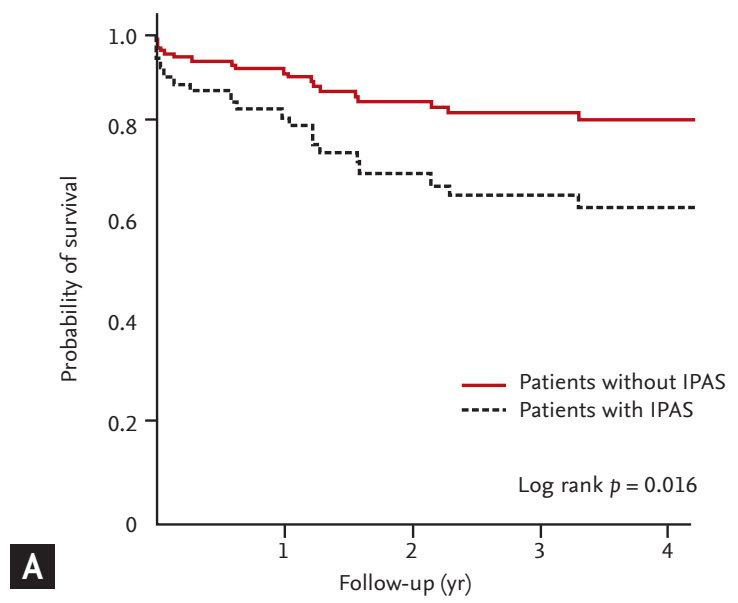

scribe the mortality. The differences between the groups were assessed using a log-rank test. A Cox proportional hazards regression was used to compute the hazard ratio (HR) as estimates for mortality. All available variables considered potentially relevant were entered into a logistic regression model: age, gender, hypertension, diabetes mellitus, dyslipidemia, smoking, family history of coronary heart disease, previous history of angina pectoris, left ventricular ejection fraction, and concomitant medications. All analyses were 2-tailed, with clinical significance defined as values of $p<0.05$.

\section{RESULTS}

\section{Clinical and angiographic characteristics}

Among 74 patients (51.4 \pm 9.9 years old, 52 males), SCD was the first clinical manifestation in 63 patients (85.1\%), whereas 11 patients (14.9\%) experienced SCD during treatment for a vasospastic angina. Forty-five patients (6o.8\%) had electrocardiographic abnormalities suggesting IPAS. Fig. 1 shows a representative patient who presented with no remarkable ECG at the initial event, though a type 1 Brugada pattern ECG was recorded 9 months later. There were no differences in baseline characteristics between the group I and II, except for a

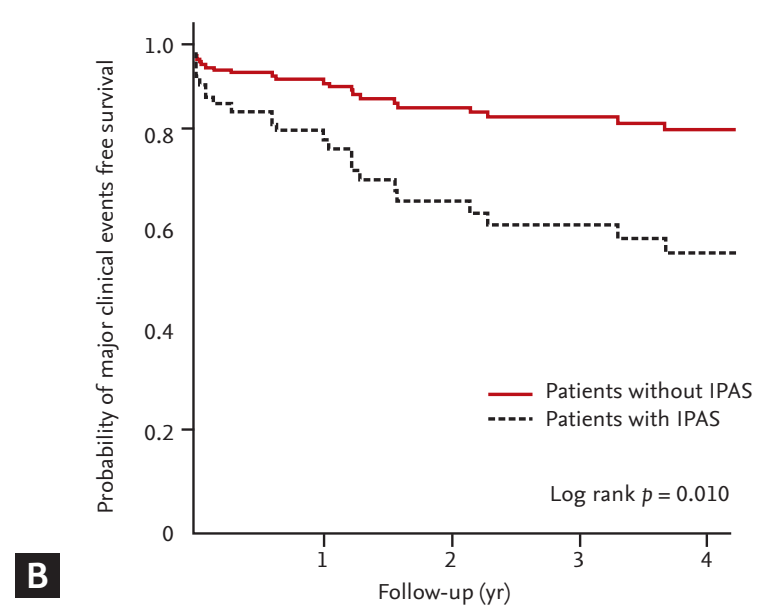

Figure 1. Masked inherited primary arrhythmia syndrome (IPAS) in a patient having coronary vasospasms. (A) No remarkable abnormality in electrocardiogram (left panel) or right coronary artery angiogram during ergonovine provocation test at the initial sudden cardiac death event. (B) Electrocardiogram 9 months later (left panel) showed type 1 Brugada pattern which was not shown at the index event, and ventricular fibrillation was induced by double ventricular extrastimuli without coronary artery spasm. 
Table 1. Baseline clinical characteristics according to the presence of inherited primary arrhythmia syndrome

\begin{tabular}{|c|c|c|c|}
\hline Characteristic & Group I $I^{\mathrm{a}}(\mathrm{n}=45)$ & Group $\mathrm{II}^{\mathrm{b}}(\mathrm{n}=29)$ & $p$ value \\
\hline Male sex & $33(73 \cdot 3)$ & $19(65 \cdot 5)$ & 0.473 \\
\hline Age, yr & $50.5 \pm 10.5$ & $53.0 \pm 8.9$ & 0.296 \\
\hline \multicolumn{4}{|l|}{ Medical history } \\
\hline Hypertension & $2(4 \cdot 4)$ & $6(20.7)$ & 0.039 \\
\hline Diabetes mellitus & $3(6.7)$ & $3(10.3)$ & 0.673 \\
\hline Dyslipidemia & 0 & $4(13.8)$ & 0.021 \\
\hline Smoking & $27(60.0)$ & $17(58.6)$ & 0.906 \\
\hline Previous history of angina pectoris & $10(22.2)$ & $10(34 \cdot 5)$ & 0.246 \\
\hline Previous history of CVA & $4(8.9)$ & 0 & 0.150 \\
\hline Previous history of CKD & 0 & o & \\
\hline Previous history of heart failure & o & 0 & \\
\hline Left ventricular ejection fraction, $\%$ & $63.9 \pm 9.7$ & $62.6 \pm 11.7$ & 0.632 \\
\hline \multicolumn{4}{|l|}{ Laboratory finding } \\
\hline Total cholesterol, ng/mL & $170.6 \pm 42.0$ & $172.0 \pm 37.7$ & 0.202 \\
\hline Triglycerides, ng/mL & $147 \cdot 6 \pm 94.1$ & $121.0 \pm 54.1$ & 0.182 \\
\hline $\mathrm{LDL}-\mathrm{C}, \mathrm{mg} / \mathrm{dL}$ & $100.7 \pm 36.6$ & $110.4 \pm 39.7$ & 0.321 \\
\hline $\mathrm{HDL}-\mathrm{C}, \mathrm{mg} / \mathrm{dL}$ & $47 \cdot 3 \pm 17 \cdot 3$ & $46.1 \pm 13.6$ & 0.775 \\
\hline Serum creatinine, mg/dL & $1.2 \pm 0.3$ & $1.1 \pm 0.4$ & 0.452 \\
\hline hsCRP, mg/dL & $0.5(0.1-1.0)$ & $0.5(0.1-3.1)$ & 0.431 \\
\hline NT-proBNP, pg/mL & $174.4(68.9-658.3)$ & $138.0(26.7-1,065.0)$ & 0.748 \\
\hline \multicolumn{4}{|l|}{ Medical treatment } \\
\hline Aspirin & $29(64 \cdot 4)$ & $19(65 \cdot 5)$ & 0.925 \\
\hline ACEI & $12(26.7)$ & $9(31.0)$ & 0.684 \\
\hline $\mathrm{ARB}$ & $9(20.0)$ & $10(34 \cdot 5)$ & 0.164 \\
\hline CCB & $37(82.2)$ & $25(86.2)$ & 0.650 \\
\hline Nitrates & $37(82.2)$ & $22(75 \cdot 9)$ & 0.506 \\
\hline Nicorandil & $22(48.9)$ & $23(79 \cdot 3)$ & 0.009 \\
\hline Statin & $21(46.7)$ & $12(41.4)$ & 0.655 \\
\hline
\end{tabular}

Values are presented as number (\%), mean $\pm \mathrm{SD}$, or median (range).

CVA, cerebrovascular accidents; CKD, chronic kidney disease; LDL-C, low density lipoprotein cholesterol; HDL-C, high density lipoprotein cholesterol; hsCRP, high sensitivity C-reactive protein; NT-proBNP, N-terminal pro-brain natriuretic peptide; ACEI, angiotensin converting enzyme inhibitor; ARB, angiotensin II receptor blocker; CCB, calcium channel blocker.

${ }^{a}$ Group I indicates patients having electrocardiographic abnormalities suggesting inherited primary arrhythmia syndrome.

${ }^{\mathrm{b}}$ Group II, patients with no electrocardiographic abnormalities suggesting inherited primary arrhythmia syndrome.

higher prevalence of hypertension and dyslipidemia in group II (Table 1). There were no significant differences in the rates of spontaneous spasms, ergonovine induced spasms, number of spastic arteries, types, and severity of spasms between groups I and II (Table 2). Eighteen patients (24.3\%) experienced recurrent SCD events despite medical therapy against coronary artery spasms.
There were no differences in baseline clinical characteristics and concomitant medications between groups III and IV (Table 3). However, group III had more diffuse and multivessel spasms than group IV (Table 3).

Electrocardiographic abnormalities suggesting IPAS Electrocardiographic abnormalities suggesting IPAS 
Table 2. Angiographic characteristics according to the presence of inherited primary arrhythmia syndromes

\begin{tabular}{|c|c|c|c|}
\hline Characteristic & Group I $I^{\mathrm{a}}(\mathrm{n}=45)$ & Group $I^{b}(n=29)$ & $p$ value \\
\hline Spontaneous spasm & $25(55.6)$ & $15(51.7)$ & 0.747 \\
\hline Ergonovine provocation test & $31(68.9)$ & $20(69.0)$ & 0.994 \\
\hline \multicolumn{4}{|l|}{ Vasospastic artery } \\
\hline Left anterior descending artery & $30(66.7)$ & $14(48.3)$ & 0.116 \\
\hline Focal & $10(33 \cdot 3)$ & $3(21.4)$ & 0.498 \\
\hline Diffuse & $17(56.7)$ & $10(71.4)$ & 0.509 \\
\hline Mixed & $3(10.0)$ & $1(7 \cdot 1)$ & 0.621 \\
\hline Total occlusion by spasm & $13(43 \cdot 3)$ & $2(14 \cdot 3)$ & 0.089 \\
\hline Left circumflex artery & $12(26.7)$ & $5(17.2)$ & 0.347 \\
\hline Focal & $1(8.3)$ & $2(40.0)$ & 0.191 \\
\hline Diffuse & $9(75.0)$ & $2(40.0)$ & 0.280 \\
\hline Mixed & $2(16.7)$ & $1(20.0)$ & 0.676 \\
\hline Total occlusion by spasm & $3(25 \cdot 0)$ & $3(60.0)$ & 0.280 \\
\hline Right coronary artery & $23(51.1)$ & $18(62.1)$ & 0.355 \\
\hline Focal & $9(39.1)$ & $10(55 \cdot 6)$ & 0.295 \\
\hline Diffuse & $12\left(5^{2.2}\right)$ & $6(33 \cdot 3)$ & 0.228 \\
\hline Mixed & $2(8.7)$ & $2(11.1)$ & 0.598 \\
\hline Total occlusion by spasm & $13(56.5)$ & $11(61.1)$ & 0.767 \\
\hline Multivessel & $14(31.1)$ & $6(20.7)$ & 0.324 \\
\hline Focal & 0 & $1(16.7)$ & 0.300 \\
\hline Diffuse & $11(78.6)$ & $4(66.7)$ & 0.573 \\
\hline Mixed & $3(21.4)$ & $1(16.7)$ & 0.657 \\
\hline Total occlusion by spasm & $4(28.6)$ & $2(33 \cdot 3)$ & 0.613 \\
\hline
\end{tabular}

Values are presented as number (\%).

${ }^{a}$ Group I indicates the patients with electrocardiographic abnormalities suggesting inherited primary arrhythmia syndromes.

${ }^{\mathrm{b}}$ Group II, the patients without electrocardiographic abnormalities suggesting inherited primary arrhythmia syndromes.

were found in 45 patients (6o.8\%): Brugada type 1 in three (4.1\%) (Fig. 1), Brugada type 2 in three (4.1\%), ARVD in three (4.1\%), LQTS in one (2.2\%), and early repolarization in 38 (51.4\%). Early repolarization included upsloping/ascending-type in 21 (28.4\%), and horizontal/descending-type in 16 (23.0\%). Two patients showed both Brugada type 1 ECG and early repolarization, whereas one patient had both LQTS and early repolarization at different points during follow-up. There were no differences in the number of ECG findings suggestive of ARVD and LQTS between groups III and IV. However, group III had a higher prevalence of Brugada type 2 ECGs and early repolarization. Notched J point elevations and horizontal/descending-type ST segments were more common in group III (Table 4).

\section{Clinical outcomes}

Mortality was 29.7\% (22 patients) during median 3.9 years (range, 2.2 to 6.5 ) of follow-up. Cardiac death occurred in 18 patients (ventricular fibrillation, $\mathrm{n}=14$; unknown, $n=4$ ), whereas non-cardiac death occurred in four patients (multi-organ failure, $\mathrm{n}=3$; malignant neoplasm, $n=1$ ). The major clinical event rate was 35.1\% (26 patients), including four patients that survived despite recurrent SCD events. Among the 18 patients having recurrent SCD events, 14 patients passed away and four patients survived.

The Kaplan-Meier estimation revealed that patients having IPAS had significantly higher mortality rates (35.6\% vs. $20.7 \%, \log$ rank $p=0.010$ ) (Fig. $2 \mathrm{~A}$ ) and major clinical event rates ( $44.4 \%$ vs. $20.7 \%, \log \operatorname{rank} p=0.010)$ 
Table 3. Clinical and angiographic characteristics according to major clinical events

\begin{tabular}{|c|c|c|c|}
\hline Characteristic & Group $\operatorname{III}^{\mathrm{a}}(\mathrm{n}=26)$ & Group IV $(\mathrm{n}=48)$ & $p$ value \\
\hline \multicolumn{4}{|l|}{ Demographic characteristics } \\
\hline Male sex & $20(76.9)$ & $32(66.7)$ & 0.357 \\
\hline Age, yr & $50.9 \pm 11.4$ & $51.7 \pm 9.1$ & 0.755 \\
\hline \multicolumn{4}{|l|}{ Medical history } \\
\hline Hypertension & $2(7 \cdot 7)$ & $6(12.5)$ & 0.705 \\
\hline Diabetes mellitus & $2(7 \cdot 7)$ & $4(8.3)$ & 0.648 \\
\hline Dyslipidemia & o & $4(8.3)$ & 0.291 \\
\hline Smoking & $16(61.5)$ & $28(58.3)$ & 0.789 \\
\hline Previous history of angina pectoris & $10(38.5)$ & $10(20.8)$ & 0.103 \\
\hline Previous history of CVA & $1(3.8)$ & $3(6.3)$ & 0.560 \\
\hline Previous history of CKD & o & o & \\
\hline Previous history of heart failure & 0 & o & \\
\hline Left ventricular ejection fraction, \% & $64.8 \pm 11.3$ & $62.7 \pm 10.2$ & 0.469 \\
\hline \multicolumn{4}{|l|}{ Medical treatment } \\
\hline Aspirin & $18(69.2)$ & $30(62.5)$ & 0.563 \\
\hline ACEI/ARB & $11(42.3)$ & $26(54.2)$ & 0.381 \\
\hline $\mathrm{CCB}$ & $19(73.1)$ & $43(89.6)$ & 0.066 \\
\hline Nitrates & $20(76.9)$ & $39(81.3)$ & 0.658 \\
\hline Nicorandil & $15(57 \cdot 7)$ & $30(62.5)$ & 0.686 \\
\hline Statin & $9(34.6)$ & $24(50.0)$ & 0.204 \\
\hline Aspirin & $13(29 \cdot 5)$ & $12(41.4)$ & 0.297 \\
\hline \multicolumn{4}{|l|}{ Angiographic characteristics } \\
\hline Spontaneous spasm & $18(69.2)$ & $22(45.8)$ & 0.054 \\
\hline Ergonovine provocation test & $17(57 \cdot 7)$ & $36(75 \cdot 0)$ & 0.125 \\
\hline \multicolumn{4}{|l|}{ Spasm type } \\
\hline Focal & $5(19.2)$ & $29(60.4)$ & 0.001 \\
\hline Diffuse & $17(65 \cdot 4)$ & $15(31.3)$ & 0.005 \\
\hline Mixed & $4(15 \cdot 4)$ & $4(8.3)$ & 0.440 \\
\hline \multicolumn{4}{|l|}{ Involved vessel } \\
\hline LAD & $15(57 \cdot 7)$ & $29(60.4)$ & 0.820 \\
\hline LCX & $9(34.6)$ & $8(16.47)$ & 0.080 \\
\hline $\mathrm{RCA}$ & $18(69.2)$ & $23(47 \cdot 9)$ & 0.078 \\
\hline Multivessel involvement & $11(42.3)$ & $9(18.8)$ & 0.029 \\
\hline Total occlusion by spasm & $12(46.2)$ & $27(56.3)$ & 0.406 \\
\hline
\end{tabular}

Values are presented as number (\%) or mean $\pm \mathrm{SD}$.

CVA, cerebrovascular accident; CKD, chronic kidney disease; ACEI, angiotensin converting enzyme inhibitor; ARB, angiotensin II receptor blocker; CCB, calcium channel blocker; LAD, left anterior descending artery; LCX, left circumflex artery; RCA, right coronary artery.

${ }^{a}$ Group III indicates the patients with major clinical events.

${ }^{\mathrm{b}}$ Group IV, the patients without major clinical events. 
Table 4. Incidence of inherited primary arrhythmia syndromes according to major clinical events

\begin{tabular}{|c|c|c|c|}
\hline Variable & Group III ${ }^{\mathrm{a}}(\mathrm{n}=26)$ & Group $\operatorname{IV}^{b}(n=48)$ & $p$ value \\
\hline Brugada ECG & $4(15 \cdot 4)$ & $2(4.2)$ & 0.068 \\
\hline Type 1 Brugada ECG & $2(7.7)$ & $1(2.1)$ & 0.502 \\
\hline Type 2 Brugada ECG & $2(7 \cdot 7)$ & $1(2.1)$ & 0.016 \\
\hline ARVD ECG & o & $3(6.3)$ & 0.578 \\
\hline Long QT syndrome & $2(7 \cdot 7)$ & o & 0.145 \\
\hline Early repolarization & $17(65 \cdot 4)$ & $21(43.8)$ & 0.033 \\
\hline \multicolumn{4}{|l|}{ J point } \\
\hline Notching & $11(42.3)$ & $11(22.9)$ & 0.026 \\
\hline Slurred & $6(23.1)$ & $10(20.8)$ & 0.798 \\
\hline \multicolumn{4}{|l|}{ ST segment pattern } \\
\hline Upsloping/ascending & $6(23.1)$ & $15(31.3)$ & 0.603 \\
\hline Horizontal & $11(42.3)$ & $6(12.5)$ & 0.001 \\
\hline \multicolumn{4}{|l|}{ Location } \\
\hline Inferior leads & $13(50.0)$ & $19(39.6)$ & 0.114 \\
\hline Lateral leads & $5(19.2)$ & $6(12.5)$ & 0.700 \\
\hline
\end{tabular}

Values are presented as number (\%).

ECG, electrocardiogram; ARVD, arrhythmogenic right ventricular dysplasia.

${ }^{\mathrm{a}}$ Group III indicates the patients with major clinical events.

${ }^{\mathrm{b}}$ Group IV, the patients without major clinical events.

(Fig. 2B) than patients without IPAS.

Patients having IPAS also had an increased risk of mortality (adjusted HR, 4.85; 95\% confidence interval [CI], 1.59 to 14.73; $p=0.005$ ), especially Brugada type (adjusted HR, 4.22; 95\% CI, 1.16 to 15.99; $p=0.034$ ) and early repolarization ECG (adjusted HR, 2.97; 95\% CI, 1.09 to 8.10; $p=0.034$ ). Among early repolarization subtypes, the horizontal/descending ST segment elevation pattern was strongly associated with increased mortality (adjusted HR, 3.00; 95\% CI, 1.23 to 7.35; $p=0.016$ ). Importantly, the use of statin (adjusted HR, 0.32; 95\% CI, 0.12 to $0.84 ; p$ $=0.020$ ) and calcium channel blocker (adjusted HR, 0.42; $95 \%$ CI, 0.07 to $0.95 ; p=0.041$ ) was seen to significantly reduce mortality after covariate adjustment (Table 5).

Eleven patients $(14.9 \%)$ received implantable cardioverter defibrillators (ICDs). The reason for ICD implantation was the recurrence of SCD events $(n=5)$, newly developed SCD events during vasospasm medication $(\mathrm{n}=1)$, inducible hemodynamically unstable ventricular tachycardia/fibrillation with electrophysiology study (EPS, $\mathrm{n}=5$ ) after the diagnosis of Brugada syndrome $(\mathrm{n}=$ 1), $\operatorname{ARVD}(n=1), \operatorname{LQTS}(n=1)$ during follow-up and without IPAS $(n=2)$. In 18 patients displaying recurrent SCD events, ICD was implanted in two patients among 14 patients who passed away, and three patients among four patients who survived. Two patients died despite ICD implantation: one patient had early repolarization, the other patient did not show any ECG abnormalities. Both passed away due to direct current shock refractory ventricular arrhythmia. The ICD implantation rate did not differ between groups I and II (17.8\% vs. 10.3\%, $p=0.511)$, or between groups III and IV (19.2\% vs. $12.5 \%, p=0.502)$.

EPS was performed in 15 patients (20.3\%), and ventricular tachyarrhythmia was induced in 11 patients (14.9\%). There were no differences in the frequency of performing EPS between groups I and II (20.0\% vs. $20.7 \%, p=$ 0.943 ), or between groups III and IV (15.4\% vs. $22.9 \%, p=$ 0.442). Similarly, there were no differences in the inducibility of ventricular tachyarrhythmia between groups I and II (50.0\% vs. 81.8\%, $p=0.604)$, or between groups III and IV $(66.7 \%$ vs. $83.3 \%, p=0.516)$.

\section{DISCUSSION}

Coronary vasospasm is a well-known cause of angina, 


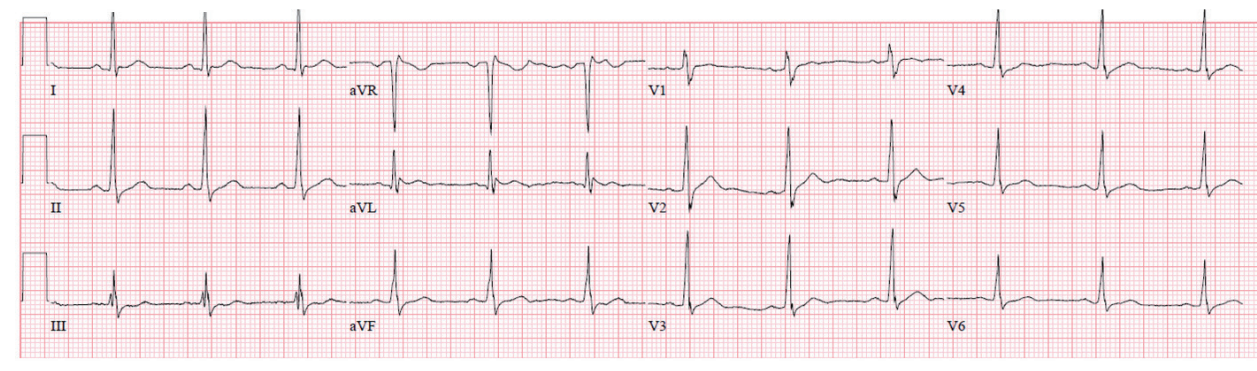

$\mathbf{A}$
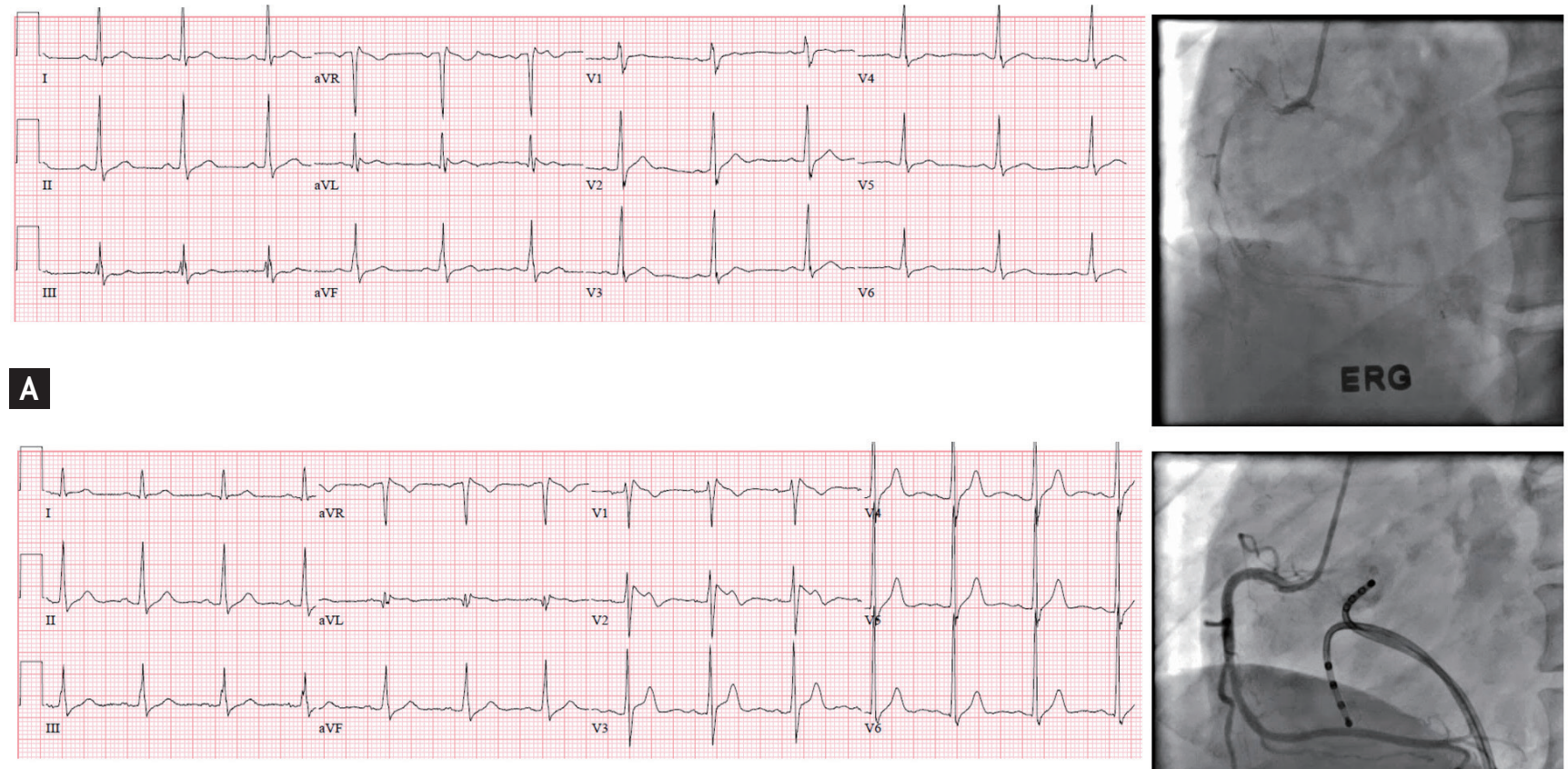

\section{B}

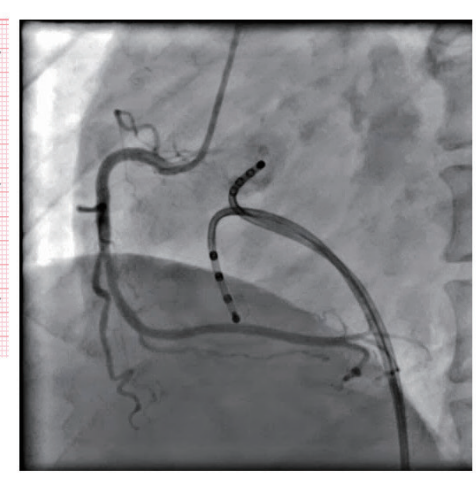

Figure 2. Kaplan-Meier survival estimation according to the presence of inherited primary arrhythmia syndrome (IPAS). (A) Patients having IPAS had significantly higher mortality (35.6\% vs. 20.7\%, log rank $p=0.010$ ) than patients without IPAS. (B) Patients having IPAS had significantly higher major clinical event rates than patients without IPAS $(44.4 \%$ vs. $20.7 \%$, log rank $p=$ o.010). ERG, ergonovine.

Table 5. Risk factors for major clinical events

\begin{tabular}{|c|c|c|c|c|}
\hline \multirow{2}{*}{ Variable } & \multicolumn{2}{|c|}{ Unadjusted HR } & \multicolumn{2}{|c|}{ Adjusted HR } \\
\hline & $95 \% \mathrm{CI}$ & $p$ value & $95 \%$ CI & $p$ value \\
\hline IPAS & $3.17(1.26-7.95)$ & 0.014 & $4.85(1.59-14.73)$ & 0.005 \\
\hline Brugada ECG & $2.61(0.89-7.63)$ & 0.079 & $4.22(1.16-15.99)$ & 0.034 \\
\hline Early repolarization & $2.36(1.04-5.35)$ & 0.039 & $2.97(1.09-8.10)$ & 0.034 \\
\hline Horizontal/descending ST segment & $3.82(1.68-8.68)$ & 0.001 & $3.00(1.23-7.35)$ & 0.016 \\
\hline Diabetes mellitus & $1.94(0.45-8.37)$ & 0.374 & $2.10(0.37-11.97)$ & 0.404 \\
\hline Hypertension & $1.87(0.42-8.25)$ & 0.410 & $1.54(0.26-9.30)$ & 0.635 \\
\hline Previous history of coronary artery disease & $1.47(0.68-3.18)$ & 0.330 & $1.91(0.87-4.20)$ & 0.106 \\
\hline Age & $1.00(0.96-1.04)$ & 0.915 & $1.02(0.98-1.06)$ & 0.437 \\
\hline Statin & $0.52(0.23-1.16)$ & 0.109 & $0.32(0.12-0.84)$ & 0.020 \\
\hline Calcium channel blocker & $0.42(0.17-0.99)$ & 0.048 & $0.25(0.07-0.95)$ & 0.041 \\
\hline
\end{tabular}

HR, hazard ratio; CI, confidence interval; IPAS, inherited primary arrhythmia syndrome; ECG, electrocardiogram.

myocardial infarction, and SCD, as fatal ventricular arrhythmia and eventual SCD can occur at the time of spasm [2]. However, it was reported that a substantial number of patients experienced lethal arrhythmia or sudden death despite being treated with appropriate medications for coronary vasospasms $[6,7]$. These findings suggest that masked hidden or unknown electrical abnormalities may play an important role in the 
development of SCD. To evaluate this assumption, we reviewed ECG abnormalities in SCD patients having spontaneous or provoked coronary vasospasms.

In a Japanese report, during an EPS, six of the 12 patients who survived out-of-hospital cardiac arrest had a both positive ergonovine provocation test and inducibility of ventricular tachyarrhythmia [15]. Parchure et al. [9] reported that QT dispersion increased in vasospastic angina patients that had complications due to cardiac arrest and syncope. These studies indicated the potential role of the concomitant electrical abnormality, but did not systemically evaluate the electrical abnormalities in SCD survivors who had coronary spasms. In the present study, a substantial number of patients (60.8\%) displayed electrical abnormalities. These results suggest that angiographic spasms are not purely a cause of SCD and that a more meticulous evaluation for IPAS is required in these patients.

\section{IPAS and coronary vasospasms}

Previous studies reported the coexistence of Brugada type ECG and coronary vasospasms [16,17]. This coexistence is particularly important in clinical practice, because the treatment strategies can be significantly different according to the diagnosis. The incidence of Brugada type ECGs has been reported in $0.9 \%$ to $11 \%$ of patients having coronary artery spasms $[18,19]$. In the present study, a substantial number of patients (60.8\%) showed electrical abnormalities; type 1 Brugada ECGs were found in three patients (4.1\%), and type 2 Brugada ECGs were found in three patients (4.1\%). The higher incidence of Brugada type ECGs in the present study may be explained by racial difference or study populations. In addition, the population of the present study included aborted SCD patients, and as such it is not surprising that the prevalence of Brugada type ECGs is higher than that of the general population.

Early repolarization patterns in ECGs have typically been considered as benign, but recent studies have reported their association with an increased risk of death $[14,20]$. Early repolarization was also reported to be associated with recurrent ventricular fibrillation in cardiac arrest survivors [21]. Rosso et al. [22] then posited that the horizontal/descending ST segment in early repolarization should be considered as "malignant," because it was more strongly correlated with idiopathic ventricular fibrillation than among all other types of early repolarization. Consistent with previous reports, in this study, patients with early repolarization were associated with an increased risk of death; specifically, early repolarization that included a horizontal/descending ST segment was more strongly associated with death. These results reconfirm that early repolarization, particularly horizontal/descending type, is closely associated with lethal ventricular arrhythmia in cardiac arrest survivors having coronary spasms. The high prevalence of Brugada type ECGs and early repolarization patterns in the present study population further suggest that not only coronary spasms but also IPAS may cause SCD.

It is possible that coronary vasospasm induced myocardial ischemia provokes or exacerbates subclinical or masked repolarization abnormalities; thereby, inducing ventricular arrhythmias. Conversely, it is also possible that IPAS might trigger coronary vasospasms and myocardial ischemia, subsequently resulting in ventricular arrhythmia. There is potential for coronary vasospasms and IPAS to have synergistic or additive effects on the development of cardiac arrest.

\section{Medical therapy, IPAS, and coronary vasospasms}

Calcium channel blockers and statins have notably improved clinical outcomes $[6,7]$. In aborted SCD patients associated with coronary vasospasms, the cessation or reduction of calcium channel blockers increased the incidences of cardiac death and non-fatal myocardial infarctions [23]. Calcium channel blockers have been an independent factor used to improve clinical outcomes. Even though a significant number of SCD patients have been associated with IPAS, the prevention of coronary artery spasms by using calcium channel blockers should be continued as an important treatment strategy.

However, microvascular ischemia can remain after the regulation of vasomotor tone by using calcium channel blockers. In addition, endothelial nitric oxide activity is reduced and endothelial function is impaired in the coronary arteries involved in spasms [24]. Myocardial scintigraphies using intravenous adenosine triphosphate with an iodinated fatty acid analogue, $15-(\mathrm{p}$ [iodine-123] iodophenyl)-3-(R,S) methylpentadecanoic acid, showed abnormal coronary spastic activity after 1 year treatment with long-acting calcium channel blockers [25]. Statins can improve vascular endothelial func- 
tion, attenuate vascular inflammation, and influence myocardial protection and remodeling [26-28]. The addition of a 3-hydroxy-3-methylglutaryl coenzyme A reductase inhibitor (fluvastatin) to conventional calcium channel blockers significantly reduced acetylcholineinduced coronary vasospasms [29]. In the present study, statins were also a strong factor that improved mortality. These findings thus suggest that IPAS are masked and hidden in a substantial number of patients, though the prevention of spasms and improvement of vascular physiology remain an important matter as well. Therefore, both statins and calcium channel blockers should be considered in the treatment of aborted SCD patients that are associated with coronary vasospasms.

\section{Study limitations}

The present study has several limitations. First, it was a retrospective analysis. Although most confounders were included in the multivariate regression analysis, it is possible that potential biases were included. Second, the sample size was relatively small. Although data were collected for over 10 years, the number of aborted SCD patients documented that had coronary spasms was limited in a single center. Therefore, a large-scale prospective multicenter trial is needed in order to clarify the relationship between the primary electrical abnormalities and clinical outcomes in SCD survivors. Third, as the follow-up duration varied in each patient, some arrhythmic events might be non-recorded. Fourth, provocation tests for IPAS were not performed systemically. Fifth, anti-spastic medications were not perfect; although there were no differences in the anti-spastic medications, patients having major clinical events showed a lower tendency to take calcium channel blockers. Therefore, well-controlled further clinical trials are needed, trials that include the provision of anti-spastic medications to all patients.

\section{KEY MESSAGE}

1. Inherited primary arrhythmia syndrome (IPAS) were not uncommon in sudden cardiac death patients having coronary vasospasms and might serve as the underlying potential for lethal complications.

2. IPAS, especially with horizontal/descending ST segment-type early repolarization and Brugada type electrocardiograms (ECGs) were associated with increased mortality and major clinical events.

3. Meticulous and careful evaluations should be considered to further elucidate hidden or subclinical IPAS in patients, including serial follow-up ECGs and electrophysiology studies.

\section{Conflict of interest}

No potential conflict of interest relevant to this article was reported.

\section{Acknowledgments}

This study was supported by grants of The Korean Society of Cardiology, The Korea Centers for Disease Control and Prevention (2013-E63005-00), and The Korean Health Technology R\&D Project ( $\mathrm{HI}_{33} \mathrm{C1}_{1527}$ ), Ministry of Health and Welfare, Republic of Korea.

\section{REFERENCES}

1. Tester DJ, Ackerman MJ. The role of molecular autopsy in unexplained sudden cardiac death. Curr Opin Cardiol 2006;21:166-172.

2. Myerburg RJ, Kessler KM, Mallon SM, et al. Life-threatening ventricular arrhythmias in patients with silent myocardial ischemia due to coronary-artery spasm. N Engl J Med 1992;326:1451-1455.

3. Moss AJ, Schwartz PJ, Crampton RS, Locati E, Carleen E. The long QT syndrome: a prospective international study. Circulation 1985;71:17-21.

4. Gaita F, Giustetto C, Bianchi F, et al. Short QT syndrome: a familial cause of sudden death. Circulation 2003;108:965-970.

5. Brugada J, Brugada R, Brugada P. Right bundle-branch block and ST-segment elevation in leads V1 through V3: a marker for sudden death in patients without demonstrable structural heart disease. Circulation 1998;97:457460.

6. Antman E, Muller J, Goldberg S, et al. Nifedipine therapy for coronary-artery spasm: experience in 127 patients. N Engl J Med 1980;302:1269-1273.

7. Kimura E, Kishida H. Treatment of variant angina with 
drugs: a survey of 11 cardiology institutes in Japan. Circulation 1981;63:844-848.

8. Suzuki M, Nishizaki M, Arita M, et al. Increased QT dispersion in patients with vasospastic angina. Circulation 1998;98:435-440.

9. Parchure N, Batchvarov V, Malik M, Camm AJ, Kaski JC. Increased QT dispersion in patients with Prinzmetal's variant angina and cardiac arrest. Cardiovasc Res 2001;50:379-385.

10. Basso C, Corrado D, Marcus FI, Nava A, Thiene G. Arrhythmogenic right ventricular cardiomyopathy. Lancet 2009;373:1289-1300.

11. Haissaguerre M, Derval N, Sacher F, et al. Sudden cardiac arrest associated with early repolarization. N Engl J Med 2008;358:2016-2023.

12. Priori SG, Wilde AA, Horie M, et al. HRS/EHRA/APHRS expert consensus statement on the diagnosis and management of patients with inherited primary arrhythmia syndromes: document endorsed by HRS, EHRA, and APHRS in May 2013 and by ACCF, AHA, PACES, and AEPC in June 2013. Heart Rhythm 2013;10:1932-1963.

13. McKenna WJ, Thiene G, Nava A, et al. Diagnosis of arrhythmogenic right ventricular dysplasia/cardiomyopathy: Task Force of the Working Group Myocardial and Pericardial Disease of the European Society of Cardiology and of the Scientific Council on Cardiomyopathies of the International Society and Federation of Cardiology. Br Heart J 1994;71:215-218.

14. Tikkanen JT, Junttila MJ, Anttonen O, et al. Early repolarization: electrocardiographic phenotypes associated with favorable long-term outcome. Circulation 2011;123:26662673 .

15. Takagi Y, Yasuda S, Takahashi J, et al. Importance of dual induction tests for coronary vasospasm and ventricular fibrillation in patients surviving out-of-hospital cardiac arrest. Circ J 2009;73:767-769.

16. Sasaki T, Niwano S, Kitano Y, Izumi T. Two cases of Brugada syndrome associated with spontaneous clinical episodes of coronary vasospasm. Intern Med 2006;45:77-80.

17. Nishizaki M, Fujii H, Ashikaga T, Yamawake N, Sakurada H, Hiraoka M. ST-T wave changes in a patient complicated with vasospastic angina and Brugada syndrome: differential responses to acetylcholine in right and left coronary artery. Heart Vessels 2008;23:201-205.

18. Noda T, Shimizu W, Taguchi A, et al. ST-segment eleva- tion and ventricular fibrillation without coronary spasm by intracoronary injection of acetylcholine and/or ergonovine maleate in patients with Brugada syndrome. J Am Coll Cardiol 2002;40:1841-1847.

19. Ong P, Bastiaenen R, Batchvarov VN, et al. Prevalence of the type 1 Brugada electrocardiogram in Caucasian patients with suspected coronary spasm. Europace 2011;13:1625-1631.

20. Gussak I, Antzelevitch C. Early repolarization syndrome: clinical characteristics and possible cellular and ionic mechanisms. J Electrocardiol 2000;33:299-309.

21. Lellouche N, Sacher F, Jorrot P, et al. Sudden cardiac arrest: ECG repolarization after resuscitation. J Cardiovasc Electrophysiol 2011;22:131-136.

22. Rosso R, Glikson E, Belhassen B, et al. Distinguishing "benign" from "malignant early repolarization": the value of the ST-segment morphology. Heart Rhythm 2012;9:225-229.

23. Takagi Y, Yasuda S, Tsunoda R, et al. Clinical characteristics and long-term prognosis of vasospastic angina patients who survived out-of-hospital cardiac arrest: multicenter registry study of the Japanese Coronary Spasm Association. Circ Arrhythm Electrophysiol 2011;4:295-302.

24. Kugiyama K, Yasue H, Okumura K, et al. Nitric oxide activity is deficient in spasm arteries of patients with coronary spastic angina. Circulation 1996;94:266-271.

25. Sueda S, Oshita A, Izoe Y, et al. A long-acting calcium antagonist over one year did not improve BMIPP myocardial scintigraphic imagings in patients with pure coronary spastic angina. Ann Nucl Med 2007;21:85-92.

26. Jain MK, Ridker PM. Anti-inflammatory effects of statins: clinical evidence and basic mechanisms. Nat Rev Drug Discov 2005;4:977-987.

27. Rosenson RS, Tangney CC. Antiatherothrombotic properties of statins: implications for cardiovascular event reduction. JAMA 1998;279:1643-1650.

28. Xu Z, Okamoto H, Akino M, Onozuka H, Matsui Y, Tsutsui $H$. Pravastatin attenuates left ventricular remodeling and diastolic dysfunction in angiotensin II-induced hypertensive mice. J Cardiovasc Pharmacol 2008;51:62-70.

29. Yasue H, Mizuno Y, Harada E, et al. Effects of a 3-hydroxy-3-methylglutaryl coenzyme A reductase inhibitor, fluvastatin, on coronary spasm after withdrawal of calcium-channel blockers. J Am Coll Cardiol 2008;51:1742-1748. 